\title{
Ghost propagator and the Coulomb form factor from the lattice
}

\author{
G. Burgio, M. Quandt, and H. Reinhardt \\ Institut für Theoretische Physik, Auf der Morgenstelle 14, 72076 Tübingen, Germany
}

\begin{abstract}
We calculate the Coulomb ghost propagator $G(|\mathbf{p}|)$ and the static Coulomb potential $V_{C}(|\mathbf{r}|)$ for $S U(2)$ Yang-Mills theory on the lattice. In view of possible scaling violations related to deviations from the Hamiltonian limit we use anisotropic lattices to improve the temporal resolution. We find that the ghost propagator is infrared enhanced with an exponent $\kappa_{\text {gh }} \gtrsim 0.5$ while the Coulomb potential exhibits a string tension larger than the Wilson string tension, $\sigma_{C} \simeq 2 \sigma$. This agrees with the Coulomb "scaling" scenario derived from the Gribov-Zwanziger confinement mechanism. PACS numbers: 11.15.Ha, 12.38.Gc, 12.38.Aw
\end{abstract}

Keywords: Coulomb gauge, Landau gauge, Kugo-Ojima, BRST, ghost, potential, Gribov 


\section{INTRODUCTION}

Yang-Mills theories in Coulomb gauge have attracted increasing interest in the last years, both in the continuum and on the lattice. Non-perturbative analytic predictions based on the Gribov-Zwanziger confinement mechanism [1, 2] turn out to be concise and elegant in this gauge and numerical simulations have confirmed them so far.

In covariant gauges the Gribov-Zwanziger approach requires a restriction of the functional integral beyond the Faddeev-Popov mechanism [3], which has long been known to be insufficient to extract a unique field representative along the gauge orbit and thus cannot be used to define the partition function beyond perturbation theory [1, 4, 5]. As a remedy one adds extra terms to the action which are, in general, non-local and often referred to as the Horizon function or Horizon condition. The purpose of these extra terms is to limit the fields in the functional integral to the so-called fundamental modular region $\Lambda$, which contains only absolute minima of the gauge fixing functional and thus eliminates the over-counting of gauge copies from the same orbit (except for topologically non-trivial copies).

In practice, however, it is almost impossible to limit the functional integral beyond the so-called first Gribov region $\Omega \supseteq \Lambda$, where the Faddeev-Popov operator is positive definite. Moreover, any restriction on the integration range imposed other than through the Faddeev-Popov mechanism will break the Becchi-Rouet-Stora-Tyutin (BRST) symmetry [6, 7]. Whether such enlarged action including Horizon terms exhibits some other symmetry and what the possible consequences in covariant gauges are (e.g. in terms of contributions from non-standard condensates [8-11]) is still under active debate, cfr. Ref. [12, 13] and references therein for recent results.

In contrast to the situation in covariant gauges sketched above, the physical implications of the Gribov-Zwanziger approach are quite transparent in Coulomb gauge. In the Hamiltonian formulation [14 17], for instance, the Gribov-Zwanziger idea amounts to a mere projection of the physical Hilbert space onto the subspace of states satisfying Gauß's law [18, 19]. Besides being conceptually simpler, this has at least two advantages:

- Unlike the Kugo-Ojima approach [20] in covariant gauges, where the existence of a globally conserved BRST charge $Q_{\mathrm{BRST}}|\Psi\rangle_{\mathrm{ph}}=0$ is essential, the Hamiltonian ap-

proach in Coulomb gauge requires no additional assumptions to ensure Gauß's law, i.e. a vanishing colour charge on physical states, $Q_{c}|\Psi\rangle_{\mathrm{ph}}=0$. 
- The construction of the physical Hilbert space in Coulomb gauge is much simpler than in the fully gauge-invariant Hamiltonian approach [21]. In fact, the consequences of Gauß's law in Coulomb gauge can be implemented exactly in a functional integral, which is therefore well suited for further approximations without the impediment of additional constraints such as the conservation of $Q_{\mathrm{BRST}}$.

The Horizon condition in Coulomb gauge implies both a static (i.e. equal-time) transverse gluon propagator [1] which vanishes at zero momentum, and a ghost dressing function which diverges in the same limit [2]. Physically, the last statement means that the Yang-Mills vacuum behaves as a perfect color dia-electric medium (i.e. a dual superconductor), because the dielectric function of the Yang-Mills vacuum agrees with the inverse ghost dressing function [22]. As a consequence the dual Meissner effect, which has long been a model for the origin of the confining force in so-called Abelian gauges, can also be applied to Coulomb gauge. A natural quantity to study confinement in Coulomb gauge is the nonAbelian Coulomb potential, which provides an upper bound for the quark-antiquark free energy $V(r) \leq V_{C}(r)$, i.e. there is no confinement without Coulomb confinement [23].

For all these reasons Coulomb gauge is best suited for direct investigations of the QCD wave functional. After pioneering analytical [24 26] and numerical work [27], such studies have experienced a broad popularity in the literature, cfr. [28 37]. In particular, the Hamiltonian approach lends itself to variational formulations [15, 17, 27], which allow to address non-perturbative problems in Yang-Mills theory in a rather direct and concise way. A main ingredient in these techniques are static (equal-time) two-point functions, so that a direct investigation of the Gribov-Zwanziger scenario for such correlators is important.

There have been a number of studies of this subject in the literature [38 41]. From previous analyses of the gluon propagator in Coulomb gauge [42 45] it is known that the difficulties in reaching the Hamiltonian limit on a lattice with a finite time-resolution can have drastic consequences: the resulting scaling violations, if untreated, prevent a multiplicative renormalization of the gluon propagator and also affect the correct analysis in the deep infrared. We therefore compute the Green functions on anisotropic lattices with a high temporal resolution and study the effects of approaching the Hamiltonian limit. We also employ a high quality of gauge fixing, particularly important for the ghost propagator.

The paper is organized as follows: in the next section, we discuss the exact definition of the relevant correlators, both in the continuum and on the lattice. We will also display the 
expectations on the conformal behaviour of the correlators in the deep-infrared, as raised by variational and functional methods. Section 3 contains a description of our numerical setup, as well as a detailed discussion of our results for both the two-point functions and the Coulomb potential. We also compare to continuum methods and give arguments to explain possible deviations. Finally, we conclude in section 4 with a brief summary and outlook.

\section{CORRELATORS IN COULOMB GAUGE}

\section{A. Correlators in the continuum}

At fixed time $t$, the Coulomb gauge condition $\nabla \cdot \mathbf{A}=0$ is complete (up to possible singularities and Gribov ambiguities, see Ref. [46]), i.e. any residual gauge symmetry is space-independent and thus acts as a global gauge at fixed time $t$. In this situation, we are interested in the static (i.e. equal time) propagators,

$$
\begin{aligned}
D(\mathbf{p}) & =\frac{\delta^{a b} \delta_{i j}}{2 N_{A}}\left\langle A_{i}^{a}(\mathbf{p}, t) A_{j}^{b}(-\mathbf{p}, t)\right\rangle=\frac{\delta^{a b} \delta_{i j}}{2 N_{A}} \int \frac{d p_{0}}{2 \pi}\left\langle A_{i}^{a}(p) A_{j}^{b}(-p)\right\rangle \\
G(\mathbf{p}) & =\frac{d(\mathbf{p})}{|\mathbf{p}|^{2}}=\frac{\delta^{a b}}{N_{A}}\left\langle\bar{c}^{a}(\mathbf{p}) c^{b}(-\mathbf{p})\right\rangle=\operatorname{tr}\left\langle(-\mathbf{D} \cdot \nabla)^{-1}\right\rangle \\
D_{0}(\mathbf{p}) & =\frac{\delta^{a b}}{N_{A}}\left\langle A_{0}^{a}(\mathbf{p}, t) A_{0}^{b}(-\mathbf{p}, t)\right\rangle \sim \\
& \sim V_{C}(\mathbf{p}) \equiv g^{2} \operatorname{tr}\left\langle(-\mathbf{D} \cdot \nabla)^{-1}\left(-\nabla^{2}\right)(-\mathbf{D} \cdot \nabla)^{-1}\right\rangle,
\end{aligned}
$$

where in the last equation contributions from non contact terms have been dropped [47]. $N_{A}$ is the dimension of the adjoint colour representation and $\operatorname{tr}$ is the normalized colour trace; we use the colour group $G=S U(2)$ with $N_{A}=3$ throughout this paper. Moreover, the covariant derivative in the adjoint representation reads $\mathbf{D}^{a b} \equiv \nabla \delta^{a b}+g \epsilon^{a b c} \mathbf{A}^{c}$, and $(-\mathbf{D} \cdot \nabla)$ is the Faddeev-Popov operator in Coulomb gauge.

Within the Hamiltonian variational approach one finds two sets of solutions for the gap equations, called "critical" [17, 48] and "subcritical" [49]. Here we will be mainly interested in the critical solution, which is also believed to be the physically relevant one. In this case one finds a Coulomb potential Eq. (4) which rises linearly as a function of the distance [48]. Furthermore, the gluon and ghost propagators in Eq. (12) exhibit a conformal scaling in 
the deep infrared, i.e. they behave as a power of momentum $|\mathbf{p}| \ll 1$,

$$
D(\mathbf{p}) \sim \frac{1}{|\mathbf{p}|^{\kappa_{\mathrm{gl}}}}, \quad d(\mathbf{p}) \sim \frac{1}{|\mathbf{p}|^{\kappa_{\mathrm{gh}}}} .
$$

Similarly, asymptotic freedom indicates that the large momentum behaviour of the propagators essentially follows from the bare action, modified by logarithmic corrections with appropriate anomalous dimensions,

$$
D(\mathbf{p}) \sim \frac{1}{|\mathbf{p}| \log ^{\gamma_{\mathrm{gl}}} \frac{|\mathbf{p}|}{m}}, \quad d(\mathbf{p}) \sim \frac{1}{\log \gamma_{\mathrm{gh}} \frac{|\mathbf{p}|}{m}} .
$$

In both regimes, the relevant exponents are further constrained by the assumption that the (static) ghost-gluon vertex is essentially trivial. i.e. proportional to the bare vertex, for any kinematical configuration. This leads directly to the so-called sum rules [49]

$$
\begin{aligned}
& \kappa_{\mathrm{gl}}+2 \kappa_{\mathrm{gh}}=1 \\
& \gamma_{\mathrm{gl}}+2 \gamma_{\mathrm{gh}}=1 .
\end{aligned}
$$

The two solutions of the variational approach are derived under the same assumption, with the specific values for the exponents [17, 48 50]:

$$
\begin{aligned}
\kappa_{\mathrm{gl}}^{c}=-1 & \kappa_{\mathrm{gh}}^{c}=1 \\
\kappa_{\mathrm{gl}}^{s} \simeq-0.6 & \kappa_{\mathrm{gh}}^{s} \simeq 0.8 \\
\gamma_{\mathrm{gl}}=0 & \gamma_{\mathrm{gh}}=\frac{1}{2}
\end{aligned}
$$

Notice that Eq. (5) implies, in general, an infrared mass generation for both gluon and ghost, unless $\kappa_{\mathrm{gl}}=1$ and $\kappa_{\mathrm{gh}}=0$, their tree-level values. Such an infrared behaviour agrees with the original analysis of Gribov [1] and, in particular, implies an infrared vanishing static gluon propagator $D(\mathbf{p}) \rightarrow 0$ and an infrared divergent ghost form factor $d(\mathbf{p}) \rightarrow \infty$, as $|\mathbf{p}| \rightarrow 0$. Physically, these findings translate into a diverging gluon self-energy $\omega(\mathbf{p}) \sim D(\mathbf{p})^{-1}$, and a vanishing dielectric function of the Yang-Mills vacuum, $\epsilon(\mathbf{p}) \sim d(\mathbf{p})^{-1}$, which in turn implies dual superconductivity [22]. In Ref. [51] the ghost and gluon propagator were also calculated from the renormalization group flow equations, assuming a bare ghost-gluon vertex and UV propagators with vanishing anomalous dimensions $\left(\gamma_{\mathrm{gl}}=\gamma_{\mathrm{gh}}=0\right)$; the corresponding IR exponents $\kappa_{\mathrm{gl}} \simeq-0.28$ and $\kappa_{\mathrm{gh}} \simeq 0.64$ turn out to be somewhat smaller than in Eq. (10).

The large momentum behaviour cited above for both propagators shows interesting nonperturbative effects as well. Despite asymptotic freedom (and in contrast to Landau gauge), 
the predictions Eq. (10) for the anomalous dimensions cannot be directly compared to perturbation theory [52, 53], since no obvious rainbow-ladder-like re-summation technique is available in Coulomb gauge. In fact, it is not even clear whether static propagators can be accessed in standard perturbation theory in the first place: the Slavnov-Taylor identities imply a highly non-trivial energy (i.e. $p_{0}$ ) dependence in the Green's functions [54], which could spoil the naive static limit and thus require a non-perturbative treatment anyhow [55/57]. Alternatively, a perturbative approach based on the functional renormalization group has been attempted in Ref. [58, 59]; their prediction $\gamma_{\mathrm{gl}}=3 / 11$ and $\gamma_{\mathrm{gh}}=4 / 11$ differs, however, quite substantially from the non-perturbative results in Eq. (10).

\section{B. Correlators on the Lattice}

A comparison between continuum and lattice Coulomb propagators was pioneered in Ref. [60]; later studies with different techniques gave, however, contradicting results [38 41]. The difficulty lies in the fact that most continuum results for static quantities are naturally obtained in the gauge $A_{0}=0$, which cannot be attained directly in lattice calculations because of the compactified time direction. Furthermore, any finite lattice has a finite time resolution and the associated discretization artifacts preclude the Hamiltonian limit and give rise to severe renormalization problems. A way to circumvent these issues was first proposed in Ref. 42 44. There it was shown that the static gluon propagator of Eq. (1) agrees, after dealing with compactification and renormalization artifacts, with the exponents in Eq. (10). More precisely, the static gluon propagator in $D=3+1$ was shown to be surprisingly well described, over the whole momentum range, by Gribov's original proposal [1]:

$$
D(\mathbf{p})=\frac{|\mathbf{p}|}{2 \sqrt{|\mathbf{p}|^{4}+M^{4}}} .
$$

As argued in Ref. [42, 43], the problem in the "naive" extraction of $D(\mathbf{p})$ from the lattice data lies in the standard, isotropic discretization of Yang-Mills theories. The use of anisotropic lattices, which approach the Hamiltonian limit [61] for large anisotropy $\xi=a_{s} / a_{t}$ (see Sec. III A), was therefore proposed as a general tool in Coulomb gauge investigations. Fur-

ther independent studies in Ref. [62, 63] have confirmed the presence of such effects and the improvement of lattice results for $\xi>1$ for the gluon correlators. Incidentally, the authors there were still not able to extract a coulomb string tension from the analysis of the temporal 
propagator, Eq. (3); the supposed equivalence between Eq. (3) and Eq. (4) [2, 23] remains therefore an open issue.

In this paper we will continue and complete the lattice analysis of Green's functions in Coulomb gauge for pure $S U(2)$ Yang-Mills theory at $T=0$, which was started in Ref. [42, 44]. We will concentrate on the ghost form factor $d(\mathbf{p})$ and the Coulomb potential $V_{C}(\mathbf{p})$, and study, in particular, the anisotropy effects and the validity of the confinement scenario sketched above. A study of the Coulomb gauge quark propagator with light dynamical fermions has been published elsewhere [45].

\section{RESULTS}

\section{A. Numerical setup}

Pure Yang-Mills theory, as any continuum field theory, can be formulated in the Hamiltonian picture [64, where space and time are treated separately. Upon discretization, simple RG-group arguments [65] indicate that for any given isotropic lattice version of the field theory in question a corresponding anisotropic counterpart lying in the same universality class exists [66]. The latter describes the same physics, albeit with two different lattice spacings $a_{s}$ and $a_{t}$ for the space and time directions. For Yang-Mills actions in $D=d+1$ dimensions built in terms of the character $\chi$ of $m \times n$ Wilson loops $P_{\mu \nu}(x ; m, n)$,

$$
S(\chi ; m, n)=\beta^{\chi} \sum_{x} \sum_{\mu>\nu=1}^{d+1}\left(1-\frac{1}{\operatorname{dim}(\chi)} \chi\left[P_{\mu \nu}(x ; m, n)\right]\right)
$$

the anisotropic counterpart will read (see Ref. [61] and references therein):

$$
\begin{aligned}
S^{\prime}(\chi ; m, n)=\sum_{x} & \left\{\beta_{s}^{\chi} \sum_{i>j=1}^{d}\left(1-\frac{1}{\operatorname{dim}(\chi)} \chi\left[P_{i j}(x ; m, n)\right]\right)\right. \\
& \left.+\beta_{t}^{\chi} \sum_{i=1}^{d}\left(1-\frac{1}{\operatorname{dim}(\chi)} \chi\left[P_{i, d+1}(x ; m, n)\right]\right)\right\} .
\end{aligned}
$$

For each choice of $\beta_{s}^{\chi} \neq \beta_{t}^{\chi}$ the two lattice spacings $a_{s}$ and $a_{t}$ have to be determined nonperturbatively. The couplings are usually parametrized as $\beta_{s}^{\chi}=\beta^{\chi} \cdot \gamma$ and $\beta_{t}^{\chi}=\beta^{\chi} \cdot \gamma^{-1}$, where $\beta^{\chi}$ is a common coupling factor, while $\gamma$ is the bare anisotropy; it is related to the true (renormalized) anisotropy $\xi=a_{s} / a_{t}$ through the renormalization constant $\eta$, i.e. we 
have $\xi=\gamma \cdot \eta$. The non-perturbative value of $\eta$ can be shown to slowly approach, in the weak coupling limit, its perturbative expression [61, 67]. In much the same fashion, the transition from an isotropic action such as eq. (12) to its anisotropic counterpart eq. (13) can be generalized to lattice actions of the form $S=\sum_{\chi, m n} c_{\chi, m n} S(\chi ; m, n)$. Such anisotropic models are very useful in lattice applications, see e.g. Ref. [61, 68] and references therein.

Turning to our specific case, we will concentrate on the standard Wilson one-plaquette action in $3+1$ dimensions, which for $S U\left(N_{c}\right)$ pure gauge theory reads:

$$
S_{W}=\sum_{x} \beta \sum_{\mu>\nu=1}^{4}\left(1-\frac{1}{N_{c}} \operatorname{Re}\left[\operatorname{Tr}\left(P_{\mu \nu}(x)\right)\right]\right)
$$

while its anisotropic version is given by:

$$
\begin{aligned}
S_{W}^{\prime} & =\sum_{x}\left\{\beta_{s} \sum_{i>j=1}^{3}\left(1-\frac{1}{N_{c}} \operatorname{Re}\left[\operatorname{Tr}\left(P_{i j}(x)\right)\right]\right)+\beta_{t} \sum_{i=1}^{3}\left(1-\frac{1}{N_{c}} \operatorname{Re}\left[\operatorname{Tr}\left(P_{i 4}(x)\right)\right]\right)\right\} \\
& =\beta \sum_{x}\left\{\gamma \sum_{i>j=1}^{3}\left(1-\frac{1}{N_{c}} \operatorname{Re}\left[\operatorname{Tr}\left(P_{i j}(x)\right)\right]\right)+\frac{1}{\gamma} \sum_{i=1}^{3}\left(1-\frac{1}{N_{c}} \operatorname{Re}\left[\operatorname{Tr}\left(P_{i 4}(x)\right)\right]\right)\right\} .
\end{aligned}
$$

The values for $a_{t}$ and $a_{s}=a_{t} \xi$ for various choices of $\beta$ and $\gamma$ have been extensively studied in the case $G=S U(3)$, see e.g. Ref. [63, 69]. For $S U(2)$ we are only aware of one study from the literature [70]. In both cases non-perturbative effects turn out to be quite large, so that the analytic one-loop calculations for $\eta$, which are in principle available for any $N_{c}$, $\beta$ and $\xi$ [61], cannot be trusted for practical applications [67, 69].

Since we will concentrate on the case of two colours, $N_{c}=2$, we have decided to redetermine the relevant parameters independently by imposing rotational invariance for the static potential extracted from space- and time-like Wilson loops [67, 69]. Our best estimates for $\gamma$ and $a_{s}$ are given in Tables $\mathrm{I}$ and $\mathrm{II}$ for a selection of values for $\beta$ and $\xi$. These results have been obtained on $L^{3} \times(\xi L)$ lattices of spacial extension up to $L=32$ and anisotropies up to $\xi=4$; we have sampled $\mathscr{O}(1000)$ configurations using a heath-bath algorithm combined with over-relaxation, which for $S U(2)$ can be easily extended to the anisotropic case. The same algorithm was also used to generate the configurations on which the measurement of Green's functions described in the next section have been performed.

The results for $\gamma$ are mostly compatible within errors with those of Ref. [70], while we find some discrepancies in the scale $a_{s}$. We have checked that our predictions for $a_{s}=a_{t}$ in the isotropic case $\xi=1$ agree with others in the literature (cfr. e.g. Ref. [71]), while 
the results of Ref. [70] are always higher than ours at the lower end of the scaling window $\beta \lesssim 2.3$. Such discrepancies could be due to different systematics inherent to the method used; higher precision might be needed to settle the issue.

\begin{tabular}{|r||c|c|c|c|c|c|c|}
\hline$\beta$ & 2.15 & 2.2 & 2.3 & 2.4 & 2.5 & 2.6 & 2.7 \\
\hline \hline$\xi=2$ & $1.654(3)$ & $1.672(3)$ & $1.712(3)$ & $1.754(4)$ & $1.796(5)$ & $1.835(6)$ & $1.870(9)$ \\
\hline$\xi=3$ & $2.375(3)$ & $2.407(3)$ & $2.474(4)$ & $2.545(4)$ & $2.608(5)$ & $2.663(7)$ & $2.710(9)$ \\
\hline$\xi=4$ & $3.106(4)$ & $3.151(4)$ & $3.243(5)$ & $3.331(5)$ & $3.406(6)$ & $3.466(7)$ & $3.511(9)$ \\
\hline
\end{tabular}

TABLE I. Bare anisotropy $\gamma$ vs. true anisotropy $\xi$, for various couplings $\beta$ in $S U(2)$.

\begin{tabular}{|c||c|c|c|c|c|c|c|}
\hline$\beta$ & 2.15 & 2.2 & 2.3 & 2.4 & 2.5 & 2.6 & 2.7 \\
\hline \hline$\xi=1$ & $1.196(6)$ & $1.061(6)$ & $0.821(6)$ & $0.616(6)$ & $0.441(5)$ & $0.290(5)$ & $0.231(5)$ \\
\hline$\xi=2$ & $1.355(7)$ & $1.206(6)$ & $0.940(6)$ & $0.711(6)$ & $0.511(5)$ & $0.338(5)$ & $0.268(5)$ \\
\hline$\xi=3$ & $1.391(7)$ & $1.239(7)$ & $0.968(6)$ & $0.732(6)$ & $0.526(5)$ & $0.345(5)$ & $0.270(5)$ \\
\hline$\xi=4$ & $1.406(8)$ & $1.254(7)$ & $0.979(6)$ & $0.739(6)$ & $0.530(5)$ & $0.346(5)$ & $0.271(5)$ \\
\hline
\end{tabular}

TABLE II. Spatial lattice spacing $a_{s}$ for the choices of $\gamma$ as in Table Ir Data are in $\mathrm{GeV}^{-1}$, assuming a Wilson string tension of $\sigma=(440 \mathrm{MeV})^{2}$. The first line gives our best estimate for the isotropic scale, cfr. e.g. Ref. [71].

It should be noticed that the scale $a_{s}$ raises with $\xi$ at a fixed coupling $\beta$, up to $\xi \gtrsim 3$ where a plateau is reached. In order to simulate at the same physical point for observables involving spatial links only (like the static correlators we are interested in), one thus needs to tune $\beta$ to $\xi$ as the latter increases.

\section{B. Coulomb gauge and the lattice Hamiltonian limit}

In Ref. [42, 43], it was shown how to quantify and treat the explicit $a_{t}$ dependence appearing in static observables after integrating their non-static counterparts w.r.t the energy $p_{0}$. However, there are still more subtle effects of the finite time resolution in lattice simulations. In Ref. 68] it was shown that the lattice Yang-Mills spectrum suffers from discretization 
effects of order $\mathscr{O}\left(a_{t}^{2}\right)$ up to $\mathscr{O}\left(a_{t}^{4}\right)$, depending on the quantum numbers at hand. Such effects are purely dynamical and linked to the rate at which the theory on an anisotropic lattice reaches the physical Hamiltonian limit $\xi \rightarrow \infty$ in which the eigenstates and the spectrum are well defined. Lattices with $\xi=5$ were eventually used in Ref. [68] to extract the masses of the physical states.

The goal of our investigation is to compare Coulomb gauge lattice results with continuum Hamiltonian calculations. Discretization effects at least of the same order as for the glueballs, if not larger, should therefore not come as a surprise. Let us illustrate this effect using the Coulomb gauge functional as an example.

To fix Coulomb gauge on each MC generated configuration we adapt the algorithm of Ref. [72, 73] as described in Ref. [42 44], i.e. we first fix one of the $N_{c}^{d}=2^{3}=8$ center flip sectors and then maximize, via a simulated annealing plus ensuing over-relaxation, separately at each time slice $t$ the gauge functional

$$
F(t)=\frac{1}{3 N_{c} L^{3}} \sum_{\mathbf{x}, i} \operatorname{Tr} U_{i}^{g}(\mathbf{x}, t), \quad U_{i}^{g}(\mathbf{x}, t)=g(\mathbf{x}, t) U_{i}(\mathbf{x}, t) g^{\dagger}(\mathbf{x}+\hat{i}, t)
$$

with respect to local gauge transformations $g(\mathbf{x}, t) \in S U(2)$. To further improve the gauge fixing quality, we start the gauge fixing engine with $n_{c}$ random gauge copies of the initial time slice (with usually $5 \leq n_{c} \leq 10$ ), and select the gauge-fixed copy with the highest value of $F(t)$. We then combine these best gauge-fixed time slices into a best gauge-fixed configuration for the flip sector chosen. Finally, we proceed to the next sector and repeat the procedure, selecting in the end the sector which gave the highest global functional $F=\sum_{t} F(t)$. Every time slice in the final gauge-fixed copy for each configuration has therefore been selected among at least $n_{c} \cdot N_{c}^{d}=40 \ldots 80$ gauge fixing runs with random starting points from all topological sectors. On top of this elaborated procedure to fix the spatial gauge freedom in each time slice separately we fix the residual temporal gauge symmetry $g(t)$ via the integrated Polyakov gauge, as described in Ref. [42 44].

As we have seen in the previous section, a constant spatial cut-off $a_{s}$ for increasing anisotropy $\xi$ can be obtained by tuning $\beta$ to $\xi ; a_{t}=\xi^{-1} a_{s}$ will then decrease linearly with $\xi^{-1}$. Naively one would then expect all observable which only depend on the spatial links to be independent of $\xi$. For instance, the Coulomb gauge functional Eq. (16) should, in principle, be a function of $a_{s}$ only, at least if our algorithm is good enough to approach the absolute minimum reliably, and if finite volume effects can be ignored [74]. This is not 
the case, as can be seen from Figure 1 the left chart displays the best value of the gauge functional $F$ from our algorithm as a function of the anisotropy $\xi$, for three fixed values of the lattice spacing $a_{s}=1.060(6), 0.556(5)$ and $0.350(5) \mathrm{GeV}^{-1}$. As the Hamiltonian limit

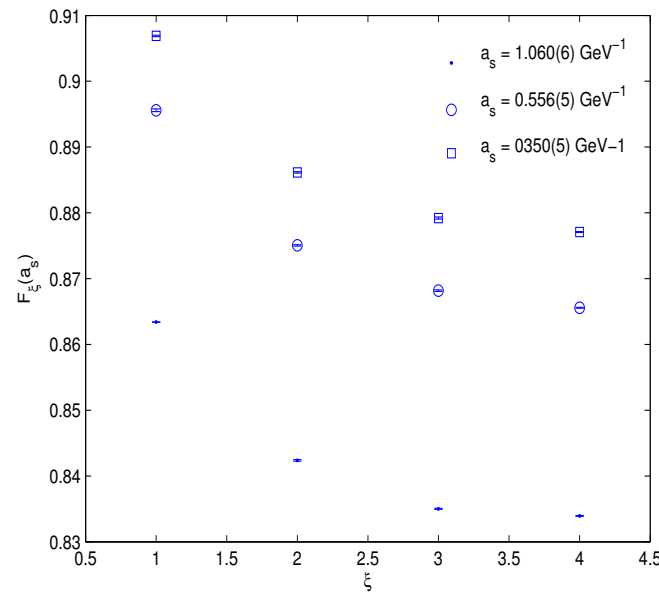

(a)

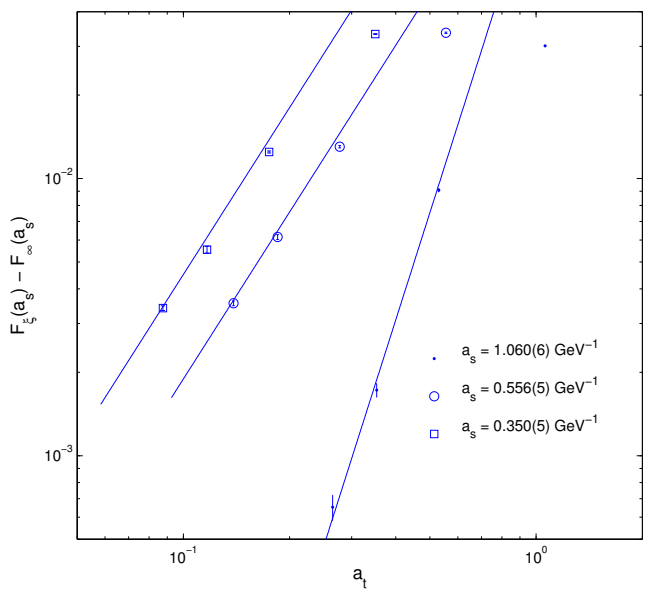

(b)

FIG. 1. (a): dependence of the gauge fixing functional $F_{\xi}\left(a_{s}\right)$ on the anisotropy $\xi$ at fixed spatial cut-off $a_{s}$. (b): Deviation of the gauge fixing functional from the Hamiltonian limit, $F_{\xi}\left(a_{s}\right)-$ $F_{\infty}\left(a_{s}\right)$, as a function of the temporal lattice spacing $a_{t}$, together with its leading power corrections.

is approached by increasing $\xi$, the functional $F$ decreases, i.e. the configurations at fixed time slice become "rougher" although $a_{s}$ is kept constant; as can be seen from the figure, the corrections with $\xi$ are several orders of magnitude higher than the Gribov noise. The leading order in the corrections to $F_{\xi}$ are well described by a power law in $\xi^{-1} \propto a_{t}$. Our best estimates for the asymptotic values $F_{\infty}$ together with the coefficient of the leading order corrections are given in Tab. III, while the right chart of Fig. 1 shows $F_{\xi}-F_{\infty}$ versus the leading correction in $a_{t}$. For the stronger couplings, this leading correction is $\mathscr{O}\left(a_{t}^{4}\right)$, while for weaker couplings we find it to scale as $\mathscr{O}\left(a_{t}^{2}\right)$.

This sensitivity to $\xi$ (or $a_{t}$ ) does not only occur in the gauge fixing functional. In Ref. [43, 62, 63] a strong dependence on the anisotropy was also seen for the temporal gluon propagator Eq. (3), although this was numerically shown to be energy (i.e. $\left.U_{0}\right)$ independent [42]. Also, preliminary results indicate that the Gribov mass $M$ in Eq. (11) slightly increases with $\xi$, saturating to a value $M \simeq 1.2(2)$ for $\xi=3-4$. In Sec. III C and IIID we will see that the ghost propagator Eq. (2) and the Coulomb potential Eq. (4), which are by 


\begin{tabular}{|c||c|c|c|}
\hline & $F_{\infty}$ & $c_{2}$ & $c_{4}$ \\
\hline \hline$a_{s}=1.060(6) \mathrm{GeV}^{-1}$ & $0.8333(2)$ & -- & $0.152(1)$ \\
\hline$a_{s}=0.556(5) \mathrm{GeV}^{-1}$ & $0.8620(2)$ & $0.058(1)$ & -- \\
\hline$a_{s}=0.350(5) \mathrm{GeV}^{-1}$ & $0.8737(2)$ & $0.055(1)$ & -- \\
\hline
\end{tabular}

TABLE III. Asymptotic extrapolation of $F_{\xi}$ together with the coefficient of the leading power correction of oder $\mathscr{O}\left(a_{t}^{4}\right)\left(a_{s}=1.060(6) \mathrm{GeV}^{-1}\right)$ and $\mathscr{O}\left(a_{t}^{2}\right)\left(a_{s}=0.556(5)\right.$ and $\left.0.350(5) \mathrm{GeV}^{-1}\right)$.

definition independent of the time-like links, both turn out to be sensitive to the anisotropy.

From Fig. 1 one can infer that lattices with $\xi \gtrsim 6$ would be needed to minimize the $a_{t}$ corrections in $F$, i.e. to reach the plateau at $F_{\infty}$. Since we also need a large spatial extension $L \simeq 50$ to explore the deep infrared momentum region, the combination of these two requirements would force us to simulate on lattices of temporal extension $N_{t} \simeq 300$, beyond the computational power at our disposal. Moreover, not all observables need to be as sensitive to $\xi$ as the gauge functional. We will therefore restrict our investigation to $\xi=1 \ldots 4$ and attempt to extrapolate to larger $\xi$ wherever necessary. Of course, a direct confirmation of our results on large lattices with high anisotropies would be highly desirable.

\section{Ghost Form Factor}

The ghost form factor in Coulomb gauge, Eq. (2), has been discussed in Refs. [38, 41, 75, 76]; neither its ultraviolet nor its infrared behavior could be determined conclusively. In the UV, the primary goal is to check the sum rule for the anomalous dimensions in Eq. (7). In the IR, the main question is whether the ghost propagator is compatible with an infrared finite behaviour, as is the case in Landau gauge [77]. If this were true, it would of course spoil the Gribov-Zwanziger mechanism and the dual superconductor argument of Ref. [22].

We calculate $d(\mathbf{p})$ by inverting the Coulomb gauge Faddeev-Popov (FP) operator through a conjugate gradient algorithm on lattices of spatial sizes up to $54^{3}$ and anisotropies up to $\xi=4$. Although for most configurations the algorithm works quite well, there are some "exceptional" cases where very small eigenvalues of the FP operator make the inversion

ill-conditioned, signaled by very bad convergence. On the other hand, we expect exactly these configurations to contribute substantially to the infrared divergence of the ghost form 
factor, since they lie close to the Gribov horizon. Our current procedure is to exclude such configurations from the Monte-Carlo ensemble; this obviously creates a bias in the data which potentially suppresses the ghost propagator at very low momenta.

Whether or not these near-horizon configurations have a measurable effect on the ghost propagator also depends on the frequency with which they appear within a MC sequence; contrary to Landau gauge [72], this seems to be relatively stable upon improvement of the gauge fixing. In our studies we have observed an ill-defined FP operator in about one of every 300 configurations, with very large uncertainties (i.e. there were also MC runs with over 1000 configurations that did not show a single near-horizon configuration). Preliminary studies of individual singular configurations with improved pre-conditioners and numerics indicate that their contribution to the ghost form factor can be enhanced by up to a factor 40 as compared to the ensemble average, again with large uncertainties due to the bad condition number. Clearly, this is a zero measure times infinite contribution problem that can only be decided by much improved statistics combined with better inversion algorithms. In this study we have not been able to resolve this issue quantitatively; the exponent which we will extract in the following must therefore be considered as a lower bound for the correct infrared behaviour.

Results for $d(\mathbf{p})$ at $\xi=1$ are shown in Fig. 2(a), while in Fig. 2(b) we give the coefficients $Z(\beta)$ needed to multiplicatively renormalize the data at different coupling, arbitrarily scaled such to let $d(\mathbf{p}) \simeq 1$ at large momentum. Although at first glance the data agree qualitatively with the above expectations, on a closer look two problems appear. First, small scaling violations can be measured within errors in the ultra-violet region; a fit to a logarithmic behavior is thus afflicted with large errors. Second, the infrared region shows two different behaviors for an intermediate momentum range $0.5 \mathrm{GeV} \lesssim|\mathbf{p}| \lesssim 1.2 \mathrm{GeV}$ and a low momentum range $0.2 \mathrm{GeV} \lesssim|\mathbf{p}| \lesssim 0.5 \mathrm{GeV}$. In the first regime the data are compatible with a power-law behavior

$$
d(|\mathbf{p}|) \propto|\mathbf{p}|^{-\kappa}
$$

with $\kappa \simeq 0.5$, while in the low-momentum region the effective $\kappa$ decreases, approaching a value $\kappa \lesssim 0.4$. By going to higher anisotropies this behavior softens and the data become more and more compatible with a stable power-law. To illustrate this effect, in Fig. 3(a) we highlight the infrared behavior showing $|\mathbf{p}|^{\kappa_{m}} d(\mathbf{p})$ for different anisotropies, where $\kappa_{m}=0.373(6)$ is the effective exponent we have measured for $L=54, \xi=1$ in the 


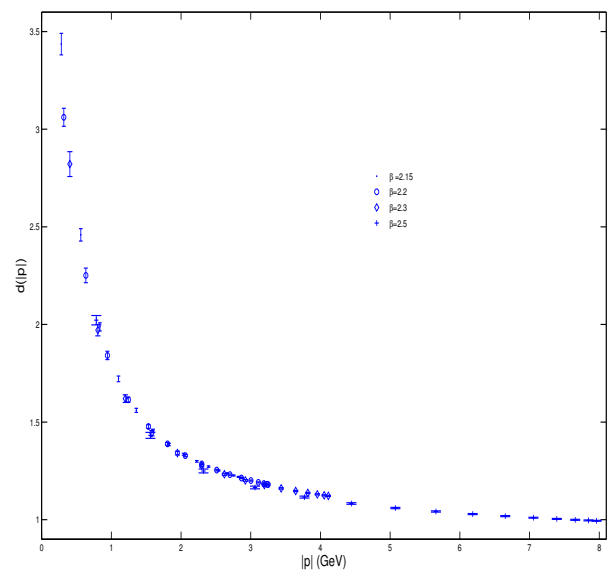

(a)

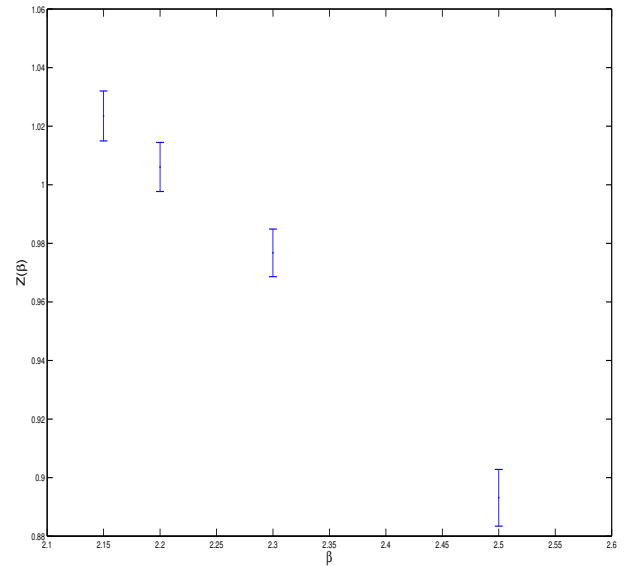

(b)

FIG. 2. (a): Ghost form factor $d(|\mathbf{p}|)$ for anisotropy $\xi=1$. (b): Renormalization coefficients for the ghost propagator, as a function of the coupling $\beta$.

lowest momentum region. The data for $\xi=1$ go to a constant while for higher anisotropies a power-law still describes the data well. In Fig. 3(b) we show the IR exponents $\kappa$ obtained from three different fitting methods for each set of data at fixed anisotropy $\xi$, i.e. temporal cut-off $a_{t}$ : the lower curve corresponds to a pure $|\mathbf{p}|^{-\kappa}$ behaviour cutting the data at $|\mathbf{p}| \lesssim 1.5 \mathrm{GeV}$; the middle one adds a linear subleading correction and leaves the same cut; the higher one combines the linear correction to the power law with a cut at $|\mathbf{p}| \lesssim 1$ $\mathrm{GeV}$. Fitting all these values of $\kappa$ to a constant $\kappa_{\mathrm{gh}}$, constrained to be the same for all three methods used, plus power law corrections in $a_{t}$ we find

$$
\kappa_{\mathrm{gh}}=0.55(1)
$$

with leading correction of order $\mathscr{O}\left(a_{t}\right)$ and a $\chi^{2} /$ d.o.f. $\simeq 0.3$.

Because of the cut on exceptional configurations discussed above we have of course introduced a bias in our data. Therefore the value in Eq. (18) can only be considered as a lower bound on the actual value of $\kappa_{\mathrm{gh}}$. Still, as far as we know, this is the first time that an IR enhanced ghost form factor can be reliably proven to exist on the lattice, making the Gribov-Zwanziger confinement scenario in Coulomb gauge fully consistent. Indeed, while in Ref. [44] it was shown that the Gribov gluon propagator Eq. (11) is IR equivalent to the massive Landau gauge solution, in the latter case lattice simulations always give an 


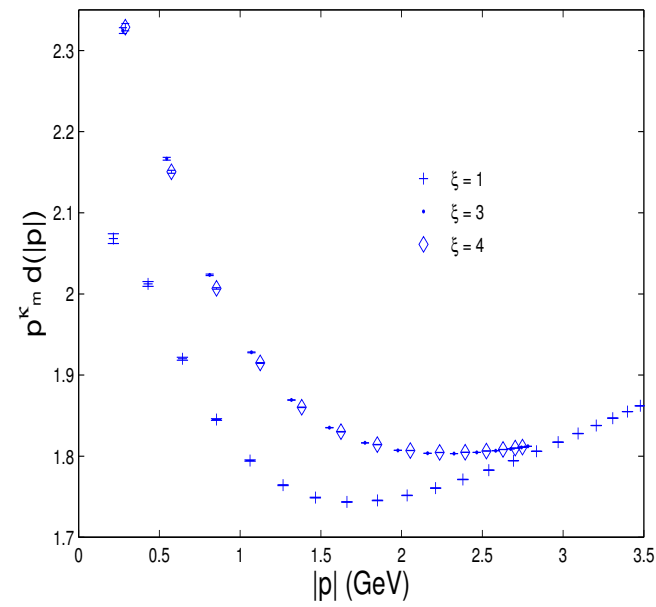

(a)

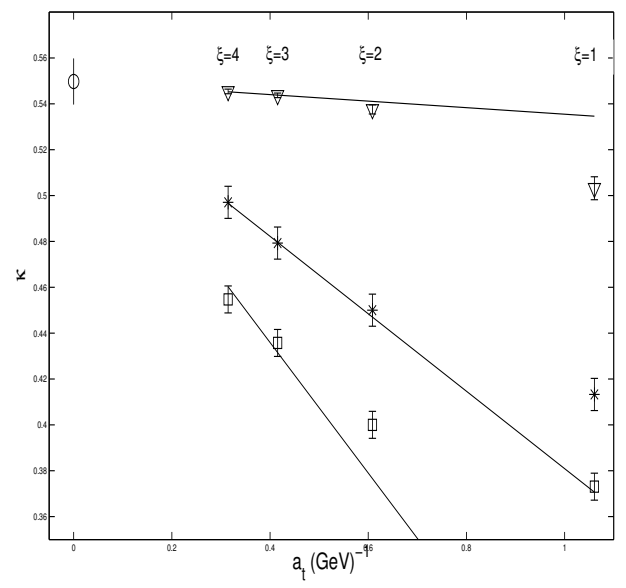

(b)

FIG. 3. (a) Infrared behavior of $|\mathbf{p}|^{\kappa_{m}} d(\mathbf{p})$ for different anisotropies $\xi$. (b) $\kappa$ extrapolation for $a_{t} \rightarrow 0$; Different symbols correspond to different fitting methods to estimates $\kappa$; points at fixed $a_{t}$ $(\xi)$ correspond to the same data set for $d(|\mathbf{p}|)$; lines give the leading correction $\propto a_{t}$ to $\kappa_{\text {gh }}$.

IR finite, i.e. tree-level like ghost form factor. How this can be made to agree with the Gribov-Zwanziger mechanism is still a debated issue.

In general, although any divergence $\left(\kappa_{\mathrm{gh}}>0\right)$ of the ghost form factor would be sufficient to support the Gribov-Zwanziger mechanism, the exact value of the infrared exponent $\kappa_{\text {gh }}$ matters for the sum rule Eq. (7), which is violated by our findings combined with the results for Gluon propagator in Refs. [42 44]. There are several possible resolutions to this issue: first, our data for the ghost propagator only covers the range down to $|\mathbf{p}| \simeq 200 \mathrm{MeV}$ with sufficient statistics and precision. Further changes in $\kappa_{\text {gh }}$ at a much smaller scale $\lambda_{I R} \ll \Lambda_{Q C D}$ can not be ruled out, although we currently do not see the onset of such deviations, while the appearance of an additional small scale in pure YM theory would raise a different kind of interpretation problem. Moreover, as we have explained above, our data for $\kappa_{\text {gh }}$ is only a lower bound since we have neglected singular configurations from near the Gribov horizon. Whether or not these configurations contribute substantially to the ghost propagator and its exponent $\kappa_{\text {gh }}$ cannot be predicted with our current computational resources. Most likely, it would also require algorithmic changes in the inversion method for ill-conditioned FP operators, and maybe even a change in the fundamental MC generator to better sample the near-horizon region in field space. 
Besides these caveats at our numerical data, the origin of the sum rule Eq. (7) itself leaves room for discussion. In essence, it is based on the assumption that (i) the gluon and ghost propagators have conformal (power-like) behaviour in the infrared and (ii) the ghost-gluon vertex receives no radiative corrections (other than an overall multiplicative renormalization) at low momenta, i.e. it is essentially bare. Both results are borrowed from Landau gauge, where Taylor's theorem gives a firm explanation why the ghost-gluon vertex is trivial as $p \rightarrow 0$. The ensuing sum rule for gluon and ghost propagator are then simple consequences, at least as long as massive solutions in the infrared can be ruled out, for which the ghost and gluon behaviour would decouple. All these assumptions are well confirmed by lattice simulations in Landau gauge.

For Coulomb gauge, however, the situation may be more involved. A careful analysis of the Slavnov-Taylor identities in Coulomb gauge exhibit a complicated interplay between transversal (spatial) and longitudinal (temporal) degrees of freedom, see Eq. (4.12) in Ref. [54]. Integrating over all energies $p_{0}$ to reach the equal-time limit could therefore induce non-trivial structure in the static Green functions, even though the proof of Taylor's theorem carries over to Coulomb gauge in the limit $|\mathbf{p}| \rightarrow 0$ at any fixed $p_{0}$. Unfortunately, no analytical calculations of the static vertex at low momenta based on the Slavnov-Taylor identities has so far been possible, and the corresponding calculation on the lattice have not been carried out. In Ref. [78], within the Hamiltonian approach, the Dyson-Schwinger equation for the ghost-gluon vertex was solved at the one loop level and little dressing was found. Furthermore, Landau gauge calculations in three (euclidian) dimensions also exhibit little dressing of the ghost-gluon vertex; it is however unclear if these results simply carry over to the four-dimensional static quantities in Coulomb gauge.

Let us now consider the ultra-violet behavior, where exceptional configurations play no role and the situation is much clearer. As mentioned above, slight scaling violations can be measured in the data for $\xi=1$. These diminish as $\xi$ is increased and the anomalous dimension of the ghost field can be determined in the Hamiltonian limit. In Fig. 4 we compare $d(\mathbf{p})$ for the lowest and highest anisotropies $\xi$. For the latter we show our best fit to the expected asymptotic behaviour with $\gamma$ given by the sum-rule in Eq. (10); the ultra-violet mass scale from the fit is $m=0.21(1) \mathrm{GeV}$, cfr. Eq. (6). 


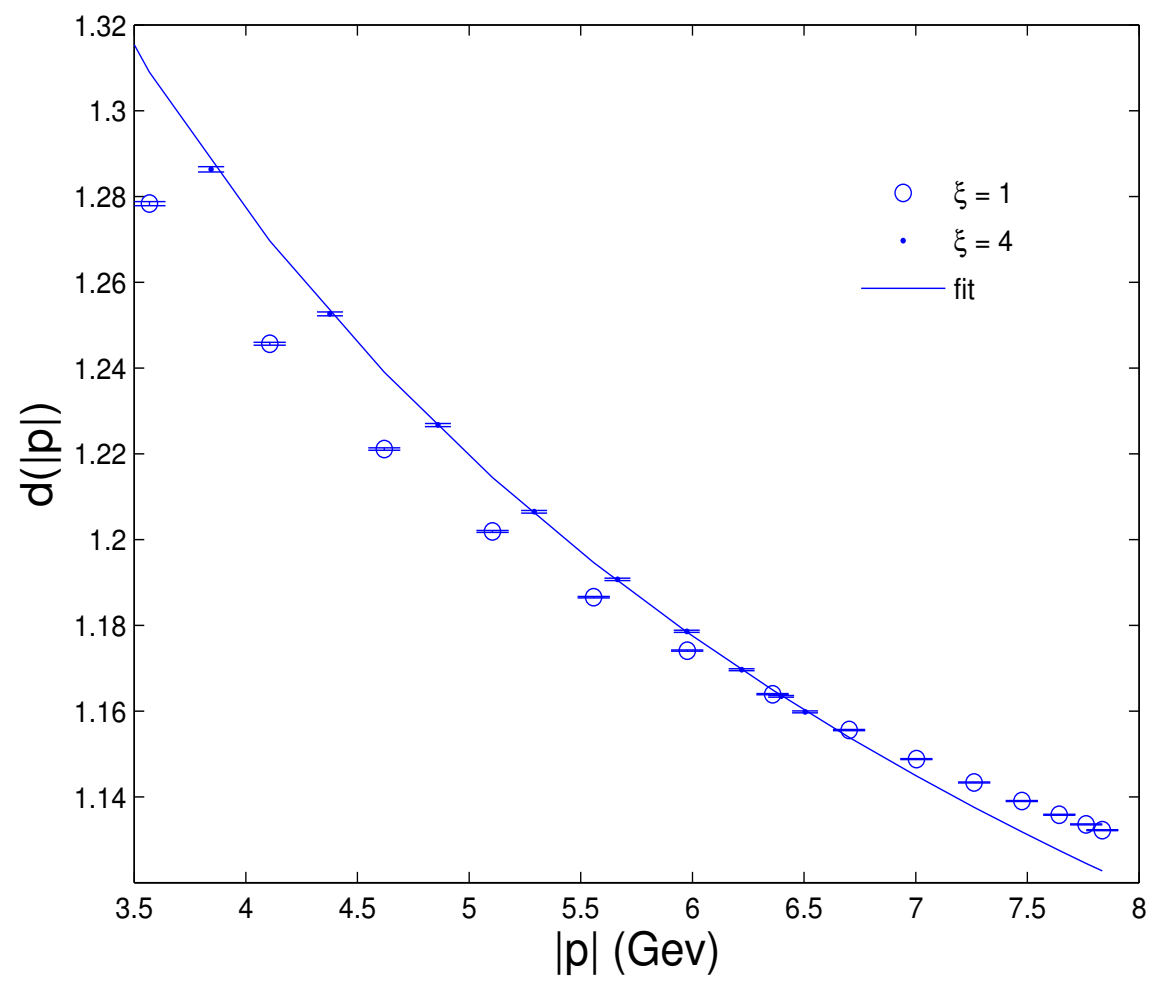

FIG. 4. UV behavior of $d^{-1}(\mathbf{p})$ for different anisotropies $\xi$ compared with Eq. 6).

\section{Coulomb Potential}

The Coulomb potential, Eq. (4), has been intensively investigated in the literature [62, 79]84. While there is general agreement that its infrared behavior is determined by a Coulomb string tension larger than the Wilson string tension [23], the value quoted for the ratio $\sigma_{C} / \sigma$ varies among the above works.

We have calculated the Coulomb potential Eq. (4) for lattices of spatial extension up to $40^{3}$ and anisotropies up to $\xi=4$. Exceptional configurations did not play a major role in this case; this may be either due to the smaller volumes and lower statistics employed, since the computation of Eq. (4) is much more expensive than Eq. (2), or to the better preconditioning due to the Laplace inversion, or both. In Fig. 5 we show the infrared behavior of $|\mathbf{p}|^{4} V_{C}(\mathbf{p}) /(8 \pi \sigma)$ for different values of $\xi$; the reason for the normalization will be clear in the following. As in the case of the ghost form factor, we observe a change in the data in the Hamiltonian limit. The corrections due to the finite temporal resolution seem to saturate at our highest values of $\xi$, although error bars are still quite large. Also, the bending down of 


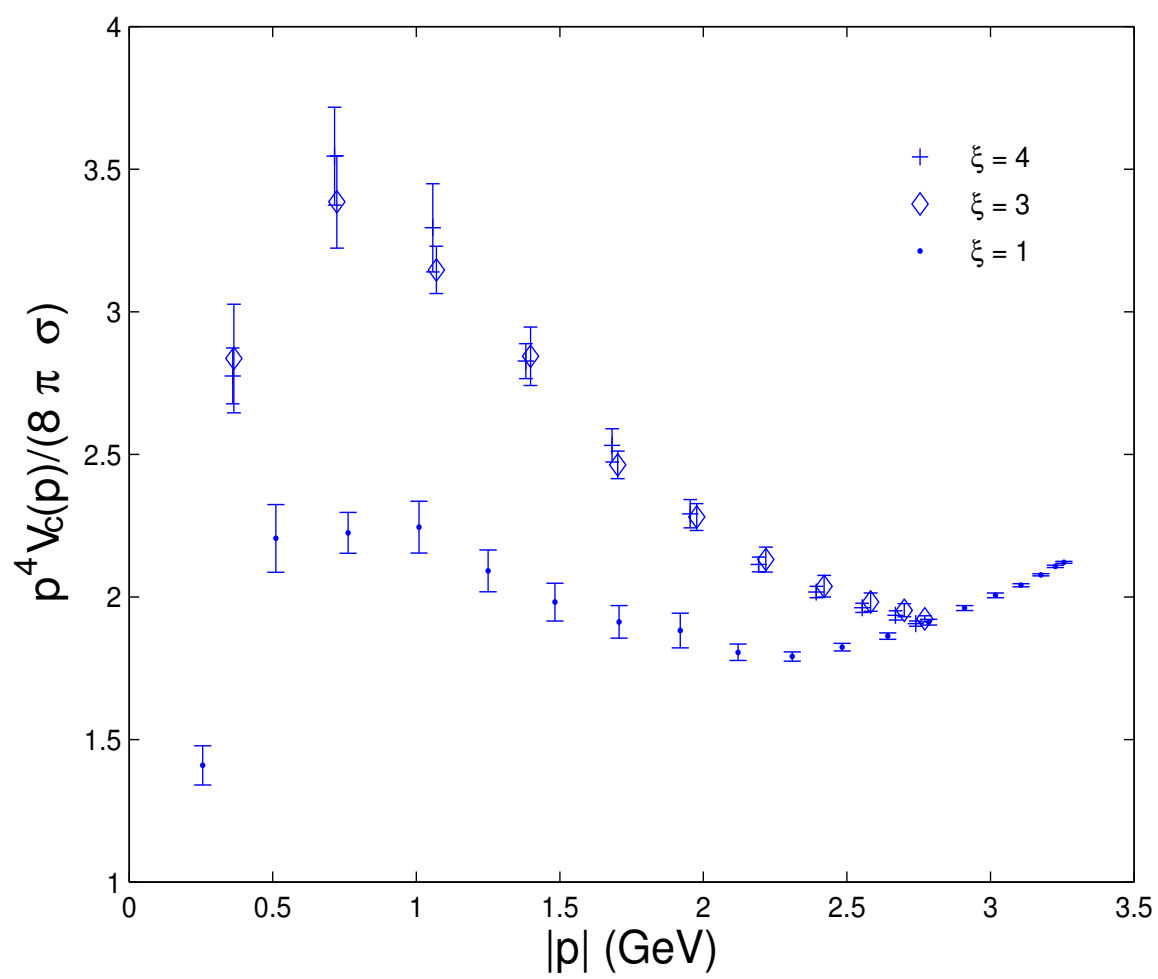

FIG. 5. Infrared behavior of $|\mathbf{p}|^{4} V_{C}(\mathbf{p}) /(8 \pi \sigma)$ for different anisotropies $\xi$.

the data at $\simeq 0.5-1 \mathrm{GeV}$, observed in all above quoted analyses for $\xi=1$, becomes milder, although it does not disappear. Its presence should indeed not come as a surprise: at least for the physical potential $V(r)$, which we know to be given by [85]

$$
V(r)=\sigma r+\mu-\frac{\pi}{12 r}+\mathscr{O}\left(\frac{1}{r^{2}}\right),
$$

we expect, after introducing a suitable IR cutoff $\lambda$ and taking $\lambda \rightarrow 0$ [86]:

$$
|\mathbf{p}|^{4} V(\mathbf{p})=8 \pi \sigma+\frac{\pi^{2}}{3}|\mathbf{p}|^{2}+\mathscr{O}\left(|\mathbf{p}|^{3}\right)
$$

since the contribution from $\mu$ in Eq. (19), which gives a term $\propto|\mathbf{p}|$ in Eq. (20), will vanish with $\lambda$. Thus $|\mathbf{p}|^{4} V(\mathbf{p})$ must have a minimum as $|\mathbf{p}| \rightarrow 0$; since its asymptotic UV behaviour is also, up to logarithmic correction, $\propto|\mathbf{p}|^{2}$, we only have two possibilities: either $|\mathbf{p}|^{4} V(\mathbf{p})$ is a monotonic function of $|\mathbf{p}|$ or, if it has some local minimum for some $|\mathbf{p}|_{m}>0$, then it must have a maximum in $0<|\mathbf{p}|_{M}<|\mathbf{p}|_{m}$.

If $V_{C}$ asymptotically behaves like $\sigma_{C} r$ the data in Fig. 5 must approach a constant as $|\mathbf{p}| \rightarrow 0$, giving direct access to the Coulomb string tension. It is natural to parametrize 
them as in Eq. (20):

$$
\frac{|\mathbf{p}|^{4} V_{C}(\mathbf{p})}{8 \pi \sigma}=\frac{\sigma_{C}}{\sigma}+\alpha|\mathbf{p}|+\gamma|\mathbf{p}|^{2}+\mathscr{O}\left(|\mathbf{p}|^{3}\right)
$$

where we would expect $\gamma>0$ and $\alpha \rightarrow 0$ in the thermodynamic limit. Fitting the data to Eq. (21) and introducing different cuts at different momenta we obtain for the Coulomb string tension

$$
\sigma_{C}=2.2(2) \sigma
$$

Notice that, even though we don't have much data in the IR region, if we optimistically constrain $\alpha \equiv 0$ we obtain a higher value for the Coulomb string tension, $\sigma_{C}=2.5(1) \sigma$. Better statistics and data from larger lattices and/or anisotropies in the low momentum region would of course be welcome to improve the result.

\section{CONCLUSIONS}

We have shown that a sufficient approach to the Hamiltonian limit, which on the lattice translates into anisotropic lattices with a high temporal resolution, is crucial for a successful investigation of the ghost form factor and the Coulomb potential. The effect of the anisotropy on static correlators lies in the different dynamics for fixed spatial lattice spacing $a_{s}$ as $a_{t}$ decreases, as we have shown in Sec. IIIB. This explains the dependence on $\xi$ found also for explicitly energy independent observables that can be evaluated in a single time slice.

In Sec. III C we have shown that the infrared exponent of the ghost form factor saturates at $\kappa_{\mathrm{gh}} \approx 0.55(1)$ when taking the Hamiltonian limit. Although our estimate may only be a lower bound due to the contribution of near-horizon configurations, the infrared divergence of the ghost form factor confirms the Gribov-Zwanziger [1, 2] confinement mechanism and the vanishing of the dielectric function for the Yang-Mills vacuum [22]. We have also confirmed quantitatively the sum rule for the anomalous dimensions of the static gluon and ghost fields in Coulomb gauge. The similar sum rule prediction in the infrared would require $\kappa_{\mathrm{gh}}=1$ in order to be compatible with the Gribov formula confirmed in ref. [44]. This is clearly violated by our best fit $\kappa_{\mathrm{gh}} \approx 0.55(1)$. We have discussed possible arguments to resolve this discrepancy, by critically analyzing both our data and the origin of the sum rule

itself. A conclusive settlement of this issue must be left to a future investigation. Finally, in Sec. IIID we have shown that the Coulomb potential is linearly rising in position space, and 
the corresponding Coulomb string tension settles nicely in the Hamiltonian limit $\xi \rightarrow \infty$, where we extract a value of $\sigma_{C} \simeq 2.2 \sigma$.

As indicated above, future investigations in this direction should concentrate on the ghost form factor and Coulomb potential on much larger spatial lattices to probe lower momenta, with improved statistics and better numerics to tackle the rare near-horizon configurations. This may help to settle the remaining question of the infrared sum rule for the exponents in the power-law of the low order Green functions.

\section{ACKNOWLEDGMENTS}

We would like to thank Peter Watson and Davide R. Campagnari for stimulating discussions. This work was partly supported by DFG under the contract DFG-Re856/6-3.

[1] V. N. Gribov, Nucl. Phys. B139, 1 (1978).

[2] D. Zwanziger, Nucl. Phys. B485, 185 (1997), hep-th/9603203.

[3] L. D. Faddeev and V. N. Popov, Phys. Lett. B25, 29 (1967).

[4] I. M. Singer, Commun. Math. Phys. 60, 7 (1978).

[5] T. P. Killingback, Phys. Lett. B138, 87 (1984).

[6] C. Becchi, A. Rouet, and R. Stora, Annals Phys. 98, 287 (1976).

[7] I. V. Tyutin (1975), 0812.0580.

[8] G. Burgio, F. Di Renzo, G. Marchesini, and E. Onofri, Phys. Lett. B422, 219 (1998), hep$\mathrm{ph} / 9706209$.

[9] R. Akhoury and V. I. Zakharov, Phys. Lett. B438, 165 (1998), hep-ph/9710487.

[10] P. Boucaud et al., JHEP 04, 006 (2000), hep-ph/0003020.

[11] D. Dudal, O. Oliveira, and N. Vandersickel (2010), 1002.2374.

[12] N. Vandersickel and D. Zwanziger (2012), 1202.1491.

[13] D. Dudal and S. P. Sorella (2012), 1205.3934.

[14] N. H. Christ and T. D. Lee, Phys. Rev. D22, 939 (1980).

[15] A. P. Szczepaniak and E. S. Swanson, Phys. Rev. D65, 025012 (2002), hep-ph/0107078.

[16] C. Feuchter and H. Reinhardt (2004), hep-th/0402106. 
[17] C. Feuchter and H. Reinhardt, Phys. Rev. D70, 105021 (2004), hep-th/0408236.

[18] H. Reinhardt and P. Watson, Phys. Rev. D79, 045013 (2009), 0808.2436.

[19] H. Reinhardt and W. Schleifenbaum, Annals Phys. 324, 735 (2009), 0809.1764.

[20] T. Kugo and I. Ojima, Prog. Theor. Phys. Suppl. 66, 1 (1979).

[21] G. Burgio, R. De Pietri, H. A. Morales-Tecotl, L. F. Urrutia, and J. D. Vergara, Nucl. Phys. B566, 547 (2000), hep-lat/9906036.

[22] H. Reinhardt, Phys. Rev. Lett. 101, 061602 (2008), 0803.0504.

[23] D. Zwanziger, Phys. Rev. Lett. 90, 102001 (2003), hep-lat/0209105.

[24] S. D. Drell, H. R. Quinn, B. Svetitsky, and M. Weinstein, Phys. Rev. D19, 619 (1979).

[25] J. P. Greensite, Nucl. Phys. B158, 469 (1979).

[26] H. Arisue, M. Kato, and T. Fujiwara, Prog. Theor. Phys. 70, 229 (1983).

[27] B. Velikson and D. Weingarten, Nucl. Phys. B249, 433 (1985).

[28] M. W. Hecht et al., Phys. Rev. D47, 285 (1993), hep-lat/9208005.

[29] I. I. Kogan and A. Kovner, Phys. Rev. D52, 3719 (1995), hep-th/9408081.

[30] D. Karabali and V. P. Nair, Nucl. Phys. B464, 135 (1996), hep-th/9510157.

[31] W. E. Brown, Int. J. Mod. Phys. A13, 5219 (1998), hep-th/9711189.

[32] W. E. Brown and I. I. Kogan, Int. J. Mod. Phys. A14, 799 (1999), hep-th/9705136.

[33] D. Karabali, C.-j. Kim, and V. P. Nair, Phys. Lett. B434, 103 (1998), hep-th/9804132.

[34] R. G. Leigh, D. Minic, and A. Yelnikov, Phys. Rev. Lett. 96, 222001 (2006), hep-th/0512111.

[35] J. Greensite and S. Olejnik, Phys. Rev. D77, 065003 (2008), 0707.2860.

[36] M. Quandt, H. Reinhardt, and G. Burgio, Phys. Rev. D81, 065016 (2010), 1001.3699.

[37] H. Reinhardt, M. Quandt, and G. Burgio, Phys.Rev. D85, 025001 (2012), 1110.2927.

[38] K. Langfeld and L. Moyaerts, Phys. Rev. D70, 074507 (2004), hep-lat/0406024.

[39] A. Voigt, E.-M. Ilgenfritz, M. Muller-Preussker, and A. Sternbeck, PoS LAT2007, 338 (2007), 0709.4585 .

[40] Y. Nakagawa, A. Nakamura, T. Saito, and H. Toki, Mod. Phys. Lett. A23, 2352 (2008).

[41] Y. Nakagawa et al., Phys. Rev. D79, 114504 (2009), 0902.4321.

[42] G. Burgio, M. Quandt, and H. Reinhardt, Phys. Rev. Lett. 102, 032002 (2009), 0807.3291.

[43] G. Burgio, M. Quandt, and H. Reinhardt, PoS CONFINEMENT8, 051 (2008), 0812.3786.

[44] G. Burgio, M. Quandt, and H. Reinhardt, Phys. Rev. D81, 074502 (2010), 0911.5101.

[45] G. Burgio, M. Schrock, H. Reinhardt, and M. Quandt (2012), 1204.0716. 
[46] R. Jackiw, I. Muzinich, and C. Rebbi, Phys. Rev. D17, 1576 (1978).

[47] D. Zwanziger, Nucl.Phys. B518, 237 (1998).

[48] D. Epple, H. Reinhardt, and W. Schleifenbaum, Phys. Rev. D75, 045011 (2007), hepth/0612241.

[49] D. Epple, H. Reinhardt, W. Schleifenbaum, and A. P. Szczepaniak, Phys. Rev. D77, 085007 (2008), 0712.3694 .

[50] W. Schleifenbaum, M. Leder, and H. Reinhardt, Phys. Rev. D73, 125019 (2006), hepth/0605115.

[51] M. Leder, J. M. Pawlowski, H. Reinhardt, and A. Weber, Phys.Rev. D83, 025010 (2011), 1006.5710.

[52] P. Watson and H. Reinhardt, Phys. Rev. D76, 125016 (2007), 0709.0140.

[53] P. Watson and H. Reinhardt, Phys. Rev. D77, 025030 (2008), 0709.3963.

[54] P. Watson and H. Reinhardt, Eur.Phys.J. C65, 567 (2010), 0812.1989.

[55] P. Watson and H. Reinhardt (2010), 1007.2583.

[56] A. Andrasi and J. C. Taylor, Annals Phys. 326, 1053 (2011), 1010.5911.

[57] A. Andrasi and J. Taylor (2012), 1204.1722.

[58] D. R. Campagnari, H. Reinhardt, and A. Weber, Phys. Rev. D80, 025005 (2009), 0904.3490.

[59] D. Campagnari, A. Weber, H. Reinhardt, F. Astorga, and W. Schleifenbaum (2009), 0910.4548 .

[60] A. Cucchieri and D. Zwanziger, Phys. Rev. D65, 014001 (2002), hep-lat/0008026.

[61] G. Burgio, A. Feo, M. J. Peardon, and S. M. Ryan (TrinLat), Phys. Rev. D67, 114502 (2003), hep-lat/0303005.

[62] Y. Nakagawa, A. Nakamura, T. Saito, and H. Toki, PoS LAT2009, 230 (2009), 0911.2550.

[63] Y. Nakagawa, A. Nakamura, T. Saito, and H. Toki, Phys.Rev. D83, 114503 (2011), 1105.6185.

[64] J. B. Kogut and L. Susskind, Phys. Rev. D11, 395 (1975).

[65] J. B. Kogut, R. B. Pearson, and J. Shigemitsu, Phys. Rev. Lett. 43, 484 (1979).

[66] B. Svetitsky and L. G. Yaffe, Nucl. Phys. B210, 423 (1982).

[67] G. Burgio, A. Feo, M. J. Peardon, and S. M. Ryan, Nucl. Phys. Proc. Suppl. 129, 396 (2004), hep-lat/0310036.

[68] C. J. Morningstar and M. J. Peardon, Phys. Rev. D60, 034509 (1999), hep-lat/9901004.

[69] T. R. Klassen, Nucl. Phys. B533, 557 (1998), hep-lat/9803010. 
[70] K. Ishiguro, T. Suzuki, and T. Yazawa, JHEP 01, 038 (2002), hep-lat/0112022.

[71] J. C. R. Bloch, A. Cucchieri, K. Langfeld, and T. Mendes, Nucl. Phys. B687, 76 (2004), hep-lat/0312036.

[72] I. L. Bogolubsky et al., Phys. Rev. D74, 034503 (2006), hep-lat/0511056.

[73] I. L. Bogolubsky et al., Phys. Rev. D77, 014504 (2008), 0707.3611.

[74] Notice that the complexity of our gauge fixing algorithm scales with the spatial volume $L^{3}$ since all time slices are fixed independently.

[75] Y. Nakagawa, A. Nakamura, T. Saito, and H. Toki, Phys. Rev. D75, 014508 (2007), heplat/0702002.

[76] G. Burgio, M. Quandt, M. Schrock, and H. Reinhardt, PoS LATTICE2010, 272 (2010), 1011.0560 .

[77] I. L. Bogolubsky, E. M. Ilgenfritz, M. Muller-Preussker, and A. Sternbeck, Phys. Lett. B676, 69 (2009), 0901.0736.

[78] D. R. Campagnari and H. Reinhardt, Phys.Lett. B707, 216 (2012), 1111.5476.

[79] J. Greensite and S. Olejnik, Phys. Rev. D67, 094503 (2003), hep-lat/0302018.

[80] A. Nakamura and T. Saito, Prog. Theor. Phys. 115, 189 (2006), hep-lat/0512042.

[81] Y. Nakagawa, T. Saito, H. Toki, and A. Nakamura, PoS LAT2006, 071 (2006), heplat/0610128.

[82] A. Voigt, E. M. Ilgenfritz, M. Muller-Preussker, and A. Sternbeck, Phys. Rev. D78, 014501 (2008), 0803.2307.

[83] Y. Nakagawa, A. Nakamura, T. Saito, and T. Toki, Mod. Phys. Lett. A23, 2348 (2008).

[84] Y. Nakagawa, A. Nakamura, T. Saito, and H. Toki, Phys. Rev. D81, 054509 (2010), 1003.4792.

[85] M. Luscher, K. Symanzik, and P. Weisz, Nucl.Phys. B173, 365 (1980).

[86] A Yukawa fall-off $e^{-\lambda r}$ is convenient to perform the Fourier integrals, but any other IR cutoff, e.g. a finite lattice size, will work as well. Of course, the two limits $\lambda \rightarrow 0$ and $|\mathbf{p}| \rightarrow 0$ do not commute; the thermodinamic limit $\lambda \rightarrow 0$ must be taken first. 